\title{
The Influence of Lighting System Photometric Characteristics on Mental State of Users. The Case Study of Keyboard Handwriting
}

\author{
Svetlana Roslyakova ${ }^{l}$, Anastasia Laushkina ${ }^{1}$, Tatiana Bragina ${ }^{1}$, Ekaterina Zemlyanova ${ }^{1}$ and \\ Oleg Basov ${ }^{l}$
}

${ }^{1}$ ITMO University, Birzhevaya line 4, St. Petersburg, 199034, Russia

\begin{abstract}
The results of existing research regarding the relationship between color temperature, lighting parameters, and mental state have limited data on multi-user space. This study is aimed at identifying the possible effect of color temperature and illuminance on mood, concentration, and, as a result, improvement or deterioration of the performance. During the experiment, the participants solved problems related to professional and educational activities. The collection of data on the mental state of the subjects was carried out automatically using an electronic survey including: self-assessment scale (SAN), state-trait anxiety inventory, visual-associative self-assessment of the emotional state of the masked personality, subjective well-being scale. In addition, to the assess performance, Kraepelin Table was used. The relationship between lighting and the mental state often manifests itself after a long-term effect. The illumination modes $3500 \mathrm{~K} 325 \mathrm{~lx}$ and $4000 \mathrm{~K} 300 \mathrm{~lx}$ are the most optimal modes for implementing artificial lighting in adaptive systems for office spaces. The collected results, conclusions, and recommendations were based on correlating the data of psychological surveys of participants and an objective method of analysis based on the investigation of the keyboard handwriting of subjects. The results show that color temperature and light levels affect behavior, mood, and performance in multiplayer environments. Taking into account the results obtained, this adaptive lighting technology can be introduced into the system of an office or study space, where adaptation is important not only for a specific person, but also for a group of users.
\end{abstract}

\section{Keywords}

Adaptive lighting system, lighting, color temperature, illuminance, performance, mental state, keyboard handwriting

\section{Introduction}

The proven effect of light on the human body causes a variety of circadian, psychophysiological, and non-visual reactions. For example, non-visual reactions include suppression of melatonin. Melatonin increases concentration and performance $[1,2,3,4,5,6]$. The photoreceptor cells of the retina are responsible for the response to light exposure; these are the cone and rod exteroceptors, as well as the cells of the retinal ganglion layer (ipRGC). ipRGCs are not directly involved in human vision, but they are associated with circadian rhythms due to a special spectral sensitivity with a maximum value at $550-555 \mathrm{~nm}$. [7, 8, 9]. Circadian rhythms refer to 24-hour cycle changes in biological processes during periods of sleep-wakefulness.

The reactions of ipRGC photoreceptors depend on such parameters of illumination as the exposure time, spectral characteristics of radiation, and level of illuminance. Noteworthy, at low illuminance levels of 10 to 15 lux and short duration of light exposure, visual photoreceptors (rods and cones) have a relatively greater contribution to non-visual responses.

GraphiCon 2021: 31st International Conference on Computer Graphics and Vision, September 27-30, 2021, Nizhny Novgorod, Russia EMAIL: svetlana.roslyakova@itmo.ru (S. Roslyakova); nastasjalausckina@mail.ru (A. Laushkina); bragina.arch@yandex.ru (T. Bragina); katya-zemlyanova95@yandex.ru (E.Zemlyanova); oobasov@mail.ru (O.Basov) ORCID: 0000-0001-6322-8668 (S. Roslyakova); 0000-0001-7887-3299 (A. Laushkina); 0000-0001-5971-8592 (T. Bragina); 0000-00028638-111X (E. Zemlyanova); 0000-0001-5788-4845 (O. Basov)

(c) (i) (c) 2021 Copyright for this paper by its authors.

Use permitted under Creative Commons License Attribution 4.0 International (CC BY 4.0).

CEUR Workshop Proceedings (CEUR-WS.org) 
Artificial lighting, as one of the many environmental factors affecting an office worker, critically impacts on the physiological and psychological functioning of the human body $[10,11,12,13]$. Incorrectly designed lighting manifests itself in a mismatch in the illuminance of the workspace, increased pulsation of the luminous flux (more than 20\%), irrelevant spectral characteristics of lighting, and uncomfortable luminance. Therefore, incorrectly selected parameters of the light environment reduce the visual comfort and well-being of workers, lead to overwork, discomfort, migraines, and insomnia [11].

The mental state of a subject responds differently to exposure to light during the day $[12,13]$. Night exposure to artificial light leads to increased anxiety and disruption of circadian rhythms. This aspect directly affects the state of performance and "flow" in the case of full involvement in an activity [8, 18]. Studies have shown the effectiveness of using lighting with a cold correlated color temperature (over $5000 \mathrm{~K}$ ) to increase concentration during the day and suppress melatonin [12, 23, 24]. Daytime exposure removes the effect of drowsiness and quickly attunes a subject to work or study $[15,16,17$, 18]. However, existing research has insufficiently characterized the influence of various spectral characteristics on the improvement of the mental state and performance. There are significant gaps in the optimal integration of the acquired knowledge into adaptive lighting systems (ALS) that adjust to the user's mental state. Usually, users have to spend much time learning how to interact with the system. Currently, the systems have no sufficient data to flexibly adapt to the expected activity of the user and their mental state. As known, the subjective concentration increases in response to a short-duration blue radiation pulse (with a maximum spectral radiation density of about $460 \mathrm{~nm}$ ) equal to 10 minutes in contrast to the long-wavelength radiation of a warm spectrum identical in duration and intensity (with a maximum spectral radiation density of about $575 \mathrm{~nm}$ ) during the day [20,21, 22].

Taking into account the interrelation of the spectral characteristics of light, circadian rhythms, and mental state, we assume it is possible to significantly influence the periods of human activity using artificial dynamic lighting. An effective impact is likely to be achieved by changing the color temperature and illuminance: neutral lighting $(\sim 4000 \mathrm{~K})$ does not have a significant effect on a subject's activity - relaxation; high temperature (more than $5000 \mathrm{~K}$ ) increases activity and productivity, reducing drowsiness; low color temperature $(2500-3000 \mathrm{~K})$ soothes the agitated state and has a regenerating effect on the body's resources [22].

Therefore, we hypothesize that changing the color temperature and illuminance level of the workspace during the day can improve the employee's overall emotional background and the work quality in multiuser spaces. The hypothesis was tested in the course of the present study.

\section{Methods}

1. Comparative analysis method:

- an overview of the ways of implementing ALS, their features and limitations.

2. The method of empirical research

- conducting an experiment aimed at obtaining data on the influence of photometric characteristics of light devices on the mental state of a subject.

3. Interdisciplinary approach

- creation of a conceptual part of the ALS and an experimental platform;

- development of a package of methods for conducting primary and secondary research for the selection on the groups of respondents together with psychologists of St. Petersburg State University;

- digital data processing:

- ANOVA, regression, and correlation analysis, using the nonparametric method of the Pearson agreement criterion;

- program software library in Python for processing keyboard handwriting data;

- development of ALS software and hardware:

- development of a hardware module for controlling lighting equipment;

- development of a software module for determining the mental state of users (control units for the level of illumination and color temperature, data collection from light sensors, $\mathrm{CO} 2$, pressure, temperature and humidity, data collection for passing psychological tests using a WEB application). 


\subsection{Participants and conditions of the experimental study}

The study involved 21 people of different genders and professional activities, the age range was from 21 to 35 years. Limitations of participation were the following: visual defects, diagnosed psychological diseases, the presence of colored or tinted contact lenses. All participants signed an informed consent to conduct the experiment and to process personal data.

Based on the analysis of the fields of activity, the following categories of subgroups were formed: programming (15 participants), design and architecture (4 participants), medicine ( 2 participants).

After the initial selection, 16 out of 21 subjects were divided into control and experimental groups based on the results of psychological testing. Each group consisted of 8 participants. At the time of the experiment, the subjects were unaware of the criteria used to be divided into groups. However, the main factor for the division into control and experimental groups was introversion and extroversion indicators.

The experiment was conducted in the scientific laboratory "Cognitive non-verbal" of the National Center for Cognitive Technologies (NCCT) of ITMO University (St. Petersburg, Birzhevaya str.16, room 408). The total duration of the experiment is from March 9 to April 2, 2021, with 1 control and 3 experimental weeks. Each week had its own schedule of light modes and one control day of the same mode (300 lux $4000 \mathrm{~K})$ - Wednesday.

The participants spent a full working day (10:00-18:00) in a coworking space designed for 8 workplaces. The subjects could solve tasks related to professional and educational activities. Data on the mental state of the subjects was collected automatically during the experiment. During the working day, the subjects passed 10-15 minute electronic questionnaires every day at 10:00, 13:00, 16:00, and 18:00.

The first group (control) was in room 408 from 10:00 to 18:00 from 09.03.21 to 12.03.21 with a lunch break (from 13:00 to 14:00). The second group (experimental) was room 408 on working days from Monday to Friday from 10:00 to 18:00 from 15.03.21 to 02.04.21 with a lunch break (from 13:00 to 14:00). In the first experimental week, during the working day, the subjects also passed 10-15-minute electronic questionnaires at control points. During the second and third experimental weeks, the conditions of the experiment and the time intervals were unchanged.

\subsection{Research protocol: light modes}

Photometric characteristics were used as an independent variable: the correlated color temperature of the lamps and the illumination level of the working area.

Based on the analysis of existing studies on the influence of changes in spectral characteristics and illumination on the human condition, the main scenarios of an adaptive lighting system were formed shown in Figure 1:

1. Standard 300 lux, $4000 \mathrm{~K}$;

2. Concentrate 1060 lux, $5800 \mathrm{~K}$;

3. Activation 675 lux, $6100 \mathrm{~K}$;

4. Relaxation 325 lux, $3500 \mathrm{~K}$;

5. Maximum relaxation 275 lux, $2700 \mathrm{~K}$.

For the control week and all control days of the same mode (Wednesday), a static neutral light mode Standard (4000 K 300 lux) was used.

In the first experimental week (from 15.03.21 to 19.03.21), the subjects performed their daily work activities under a given lighting mode Activation (6100 K 675 lux).

In the second experimental week (from 22.03.21 to 26.03.21), the Maximum relaxation light mode (2700 K 275 lux) was introduced.

The third experimental week (from 29.03.21 to 02.04.21) became a platform for testing dynamic lighting and studying its influence in comparison to the static modes. Therefore, a mode with dynamic lighting was installed according to this schedule:

Monday - 10:00-13:00 - Relaxation 3500 K 325 lux, 13:00 - 14:00 - Standard, 14:00-18:00 Activation $6100 \mathrm{~K} 675$ lux. 
Tuesday - 10:00-13:00 - Activation 6100 K 675 lux, 13:00 - 14:00 - Standard, 14:00-18:00 Relaxation 3500 K 325 lux.

Thursday - 10:00-13:00 - Relaxation 3500 K 325 lux, 13:00 - 14:00 - Standard, 14:00-18:00 Concentrate $5800 \mathrm{~K} 1060$ lux.

Friday - 10: 00-13:00 - Concentrate 5800 K 1060 lux, 13:00 - 14:00 - Standard, 14:00-18:00 Relaxation 3500 K 325 lux.

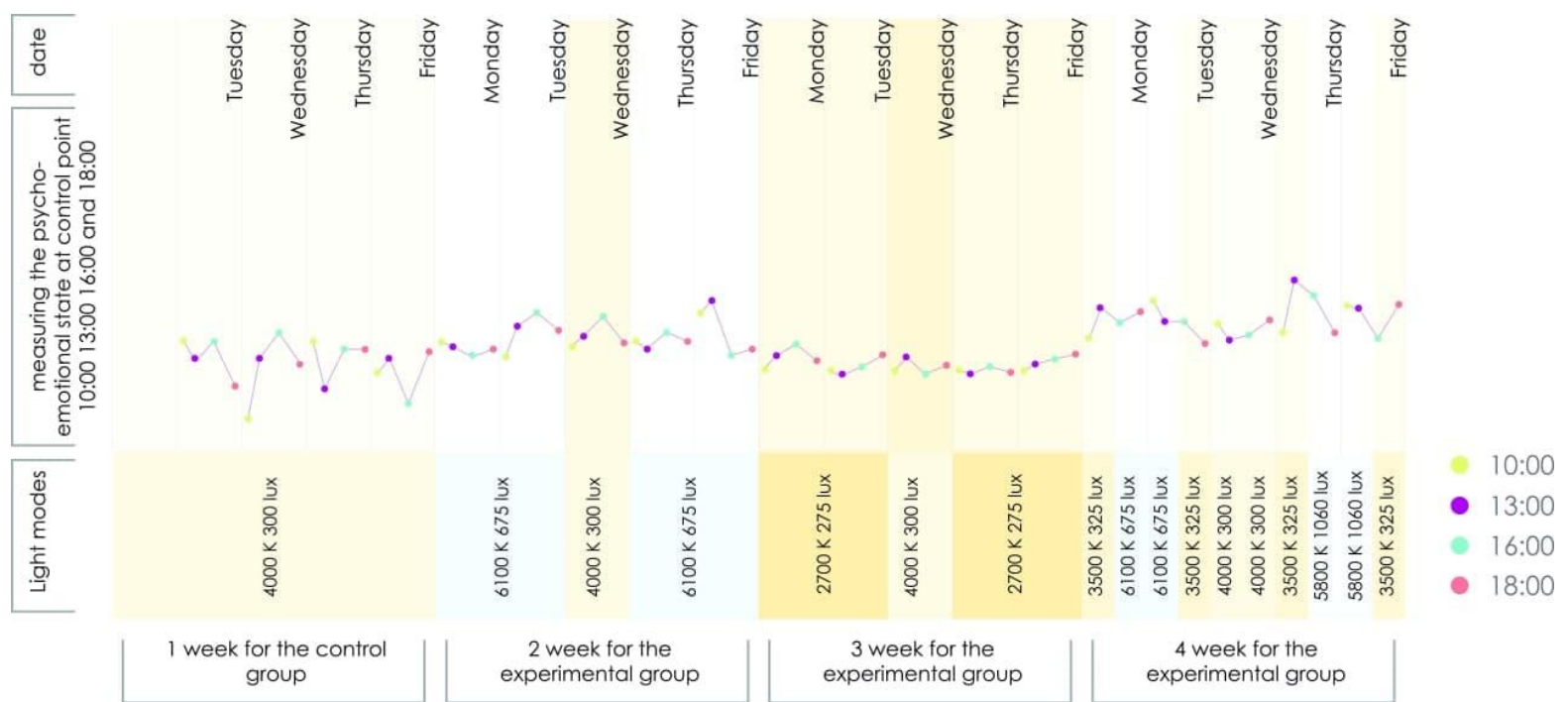

Figure 1: Temporal distribution of light modes and mental state for the control and experimental groups (1-4 weeks of the experiment)

\subsection{Study protocol: stress factors}

During the working day, to create a stressful situation, stress factors were introduced into the working field of the subjects: noise and cognitive tasks with a time limit. Stress factors for the two groups were implemented in the same format: for 15 minutes 3 times per day. The time of introduction of the stress factors was determined randomly. Frequency of the stress factors introduction was the following: morning stress factor (interval 10:00-13:00) - blue or purple noise , lunch stress factor (interval 14:00-16:00) - tasks for the allotted time, evening stress factor (interval 16:00 - 18:00, time for stress factor) - blue or purple noise.

\subsection{Methods for determining the mental state of participants}

To track the change in the mental state of the experiment participants in the conditions of an office day, a package of diagnostic methods was developed in consort with a group of psychologists of the University of St. Petersburg State University. Currently, more and more users' workplace is equipped with a computer. Therefore, data on changes in the behavior and stress state in a group of subjects were taken using the characteristics of the keyboard handwriting, as well as using subjective data about the state of a person. These data include the results of electronic questionnaires related to the current psycho-emotional state: visual-associative self-assessment of the personal emotional state, sleep assessment scales, and the subjective well-being scale. The technique was developed by N. P. Fetiskin [27], and it is intended for express diagnostics of several emotional states based on the choice of reference masks that correspond, in the opinion of the subject, to their current state. The sleep assessment scales demonstrate the dynamics of the sleep quality and quantity during the experiment. The subjective well-being scale allows to record the degree of lighting influence on working performance.

When interpreting the test responses (sleep assessment scale, insomnia severity index (insomnia), visual-associative self-assessment of the emotional state of the subject, subjective well-being scale), an integral indicator is calculated for the entire study period and the dynamics of changes is analyzed. 
The method of assessing the psychoemotional condition using the characteristics of keyboard handwriting is harmless and painless for subjects, and its correlation with the survey data allows avoiding false conclusions in the interpretation of the study results.

To implement the task of noninvasive objective data collection on the mental state of the experimental participants, the program for collecting and analyzing keyboard handwriting information calculates such parameters as:

- $\quad$ input dynamics (the time between keystrokes and the time they are held);

- $\quad$ input speed (the result of dividing the number of characters by the printing time);

- the number of overlaps between the keys;

- $\quad$ using the keys to print capital letters;

- the frequency of errors when entering.

To implement the task of non-invasive subjective data collection on the mental state of the experiment participants, a web application was developed with access to the survey material and an intuitive interface. The Telegram chatbot daily sent a reminder link at a fixed time.

\section{Results}

Apps collected data on assessing the influence of lighting on the mental state were compiled using keyboard handwriting and a package of psychological techniques. The data was collected continuously for four weeks every working day.

The results of the control and experimental groups were qualitatively and quantitatively compared according to the indicators of psychological tests between 1st and 2nd weeks and the assessment of $2 \mathrm{nd}$, $3 \mathrm{rd}$ and 4th weeks; the comparison was pairwise. The results mean that 1 st week is an investigation of the control group, 2nd, 3rd and 4th - of the experimental group. The results of this study describe only statistically significant results.

\subsection{Results of the analysis of subjective web questionnaires}

\subsubsection{Comparison of 1 week (control group) and 2 week (experimental group)}

Performance is one of the most critical indicators of a person in an office or educational space. This study revealed that the working performance in the experimental and control groups is different (Figure 2). Performance on Wednesday in the control group ( 2 week) increased by 0.089 compared with the control group's working capacity ( 1 week). The deviation from the background value of the performance indicator was +0.044 . It means a positive dynamics of the change in performance in the mode of 6100 K 675 lux.

Lighting activated the subjects at 18:00 on Wednesday (according to a subjective assessment) more in the experimental group ( 2 week) than in the control group (1 week) (Figure 3 ). These data were obtained during a subjective assessment of lighting. This indicator had a positive trend when compared with the background value (an increase of 11,687).

It is also worth noting that sleep is essential for a subject's well-being in addition to productivity. It is the sleep that affects a subject's mood, concentration and health in the long term. The quality of sleep in the $6100 \mathrm{~K} 675 \mathrm{~lx}$ mode is lower compared with the $4000 \mathrm{~K} 300 \mathrm{~lx}$ mode $(-0,625)$ (Figure 4). At the same time, the quality of sleep at $4000 \mathrm{~K} 300$ lux increased if compared with background measurements $(+0,312)$. This fact shows a positive effect of the light mode of $4000 \mathrm{~K} 300$ lux on the quality of sleep of the subjects. 


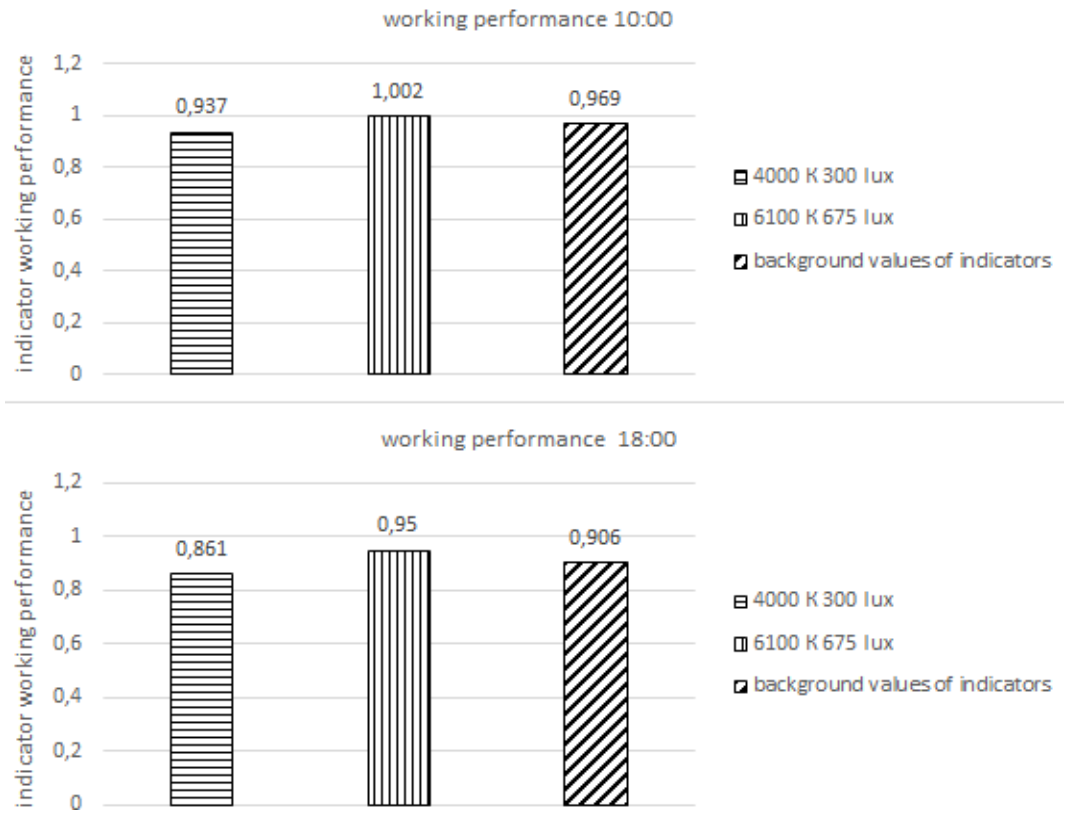

Figure 2: Performance the control and experimental groups on Wednesday at 10:00 and 18:00

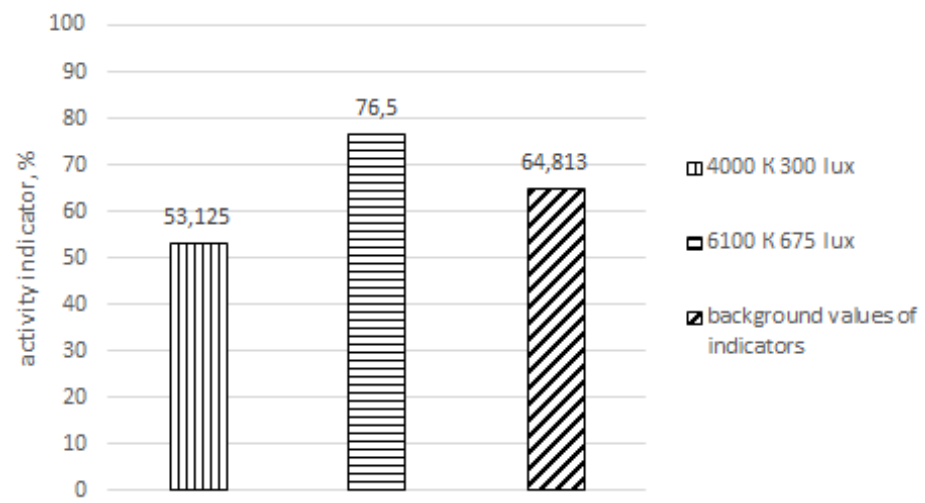

Figure 3: Distribution of self-assessment "lighting activates-lighting relaxes" in the control and experimental groups on Thursday

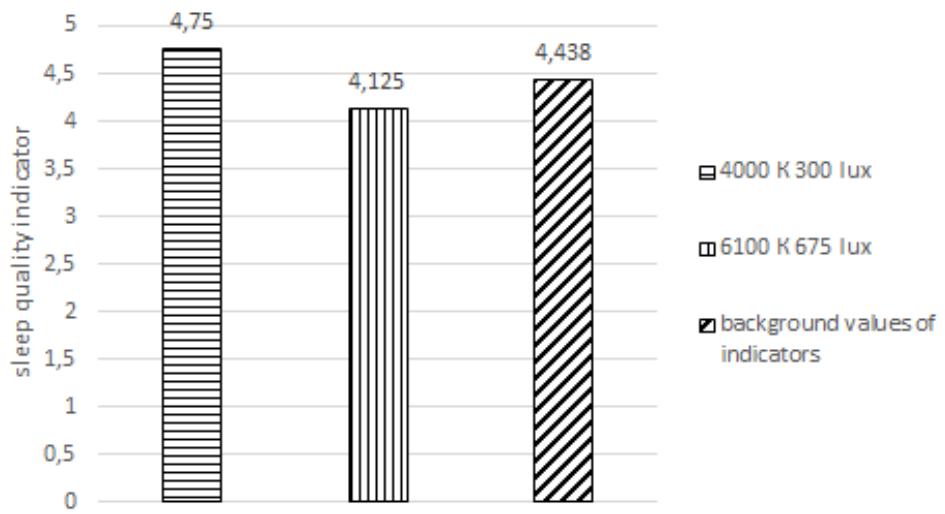

Figure 4: Sleep quality indicator in the control and experimental groups on Thursday

A good mood can reduce the rate of overwork of an employee and increase resistance to stress at work. This indicator was also studied when changing the light modes. The mood indicator in the control group 1 week was higher compared with the experimental group 2 weeks (an increase of 0.9) of the measurement on Thursday. The level of deviation from the background value of the indicator was +0.45 . This result indicates a positive dynamics of the influence of the light mode of $4000 \mathrm{~K} 300$ lux on mood. 
The indicator of the overwork degree for a comprehensive study of lighting was also considered. The main reason for overwork is the discrepancy between the duration and severity of work and rest time. In addition, a factor of overwork development can be an unsatisfactory working environment. The indicator of overwork in the experimental group tends to increase. The degree of overwork in the experimental group ( 2 week) is higher than the indicator of the control week $(1$ week) $(+5.25)$ (Figure $5)$. The indicator of overwork is higher in the control group ( 2 week) compared with background measurements $(+2.625)$. It can be concluded that there was a minimum degree of overwork in the control group ( 1 week). In the experimental group ( 2 week), this level was within the average values range.

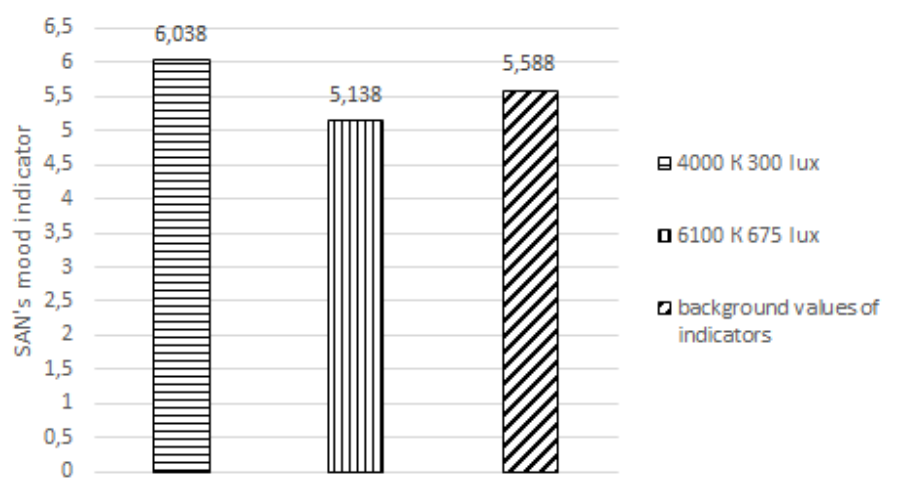

Figure 5: SAN's mood indicator in the control and experimental groups on Thursday

\subsubsection{Comparison of 1 week (control group) and 2 week (experimental group)}

Initially, we considered the subjective assessment of the overall lighting when comparing three weeks with different modes but with the same subjects. Thus, the subjects rated the light mode of 6100 K 675 lux as the most activating - 80\%, and the most relaxing mode was $2700 \mathrm{~K} 275$ lux - 40\% (measurements at 18:00). The performance indicators at 10:00 and 18:00 on Thursday in the 2nd, 3rd, 4th weeks are shown in Figure 6.

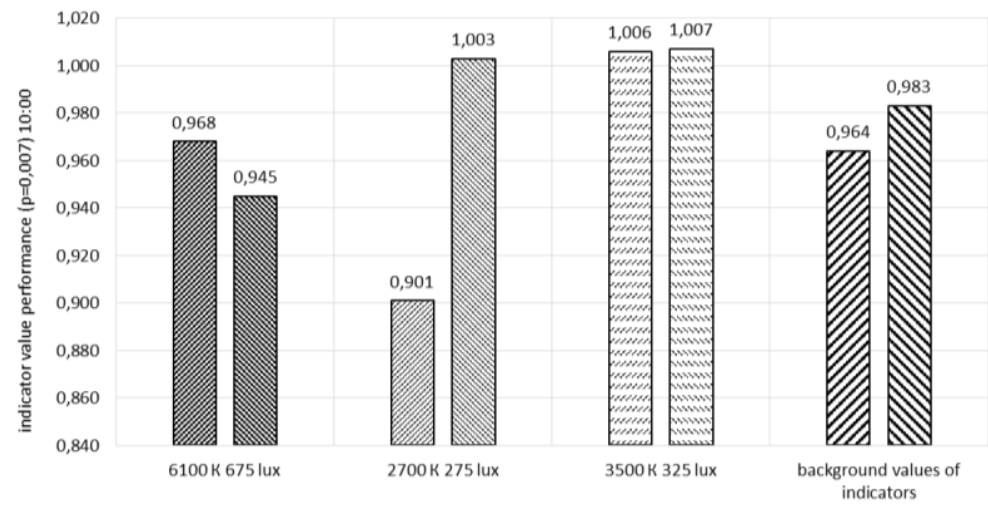

Figure 6: Results of performance indicators of the experimental group on Thursday of the 1st, 2nd and 3rd experimental weeks

A decrease in the level of performance by the end of the day was revealed in 2 weeks $(6100 \mathrm{~K} 675$ lx) of the study. At the same time, the activity level by the end of the day had the highest value. In the 3 week ( $2700 \mathrm{~K} 275 \mathrm{~lx})$, there was a slight decrease in performance at the beginning of the day. By the end of the day, the level of performance increased, but at the same time, the level of activity decreased. A decrease in the level of activity is also observed in the 4th experimental week, but the level of efficiency had high indicators during morning and evening measurements. This result may be correct because the afternoon mode was $5800 \mathrm{~K} 1060$ lux, which positively affects the concentration of the subjects. 
In general, it appeared that the activating mode was $6100 \mathrm{~K} 675$ lux. The mode of $2700 \mathrm{~K} 275$ lux was the least comfortable for the subjects. The lighting modes of $3500 \mathrm{~K} 325$ lux and $4000 \mathrm{~K} 300$ lux were the most optimal modes.

\subsection{Results of the analysis of keyboard handwriting}

The program for evaluating keyboard handwriting recorded data on the keystrokes on the keyboard. Then the following parameters were calculated:

- $\quad$ input dynamics (the time between keystrokes and the time they are held down);

- $\quad$ input speed (the result of dividing the number of characters by the print time);

- number of overlaps between keys;

- $\quad$ using keys to print capital letters;

- frequency of input errors;

- $\quad$ quantity of symbols.

Table 1 shows data on the average percentage distribution of the number of characters and the number of errors on Mondays during the experiment.

Table 1

Distribution of the number of characters and the number of errors on Mondays

\begin{tabular}{|c|c|c|c|c|c|c|c|c|}
\hline \multicolumn{9}{|c|}{ Week - lighting mode } \\
\hline & \multicolumn{2}{|c|}{$\begin{array}{c}1 \text { week - } 4000 \text { K } 300 \\
\text { lux }\end{array}$} & \multicolumn{2}{|c|}{$\begin{array}{c}2 \text { week - } 6100 \mathrm{~K} \\
675 \mathrm{lux}\end{array}$} & \multicolumn{2}{|c|}{$\begin{array}{c}3 \text { week - } 2700 \mathrm{~K} \\
275 \text { lux }\end{array}$} & \multicolumn{2}{|c|}{$\begin{array}{c}4 \text { week - } 3500 \mathrm{~K} \\
325 \text { lux, } 6100 \mathrm{~K} 675 \\
\text { lux }\end{array}$} \\
\hline time & \multicolumn{8}{|c|}{$\%$ of number of characters per day } \\
\hline $\begin{array}{l}10: 00- \\
11: 00\end{array}$ & 18,6 & 15,7 & 22,9 & 16,3 & 10,6 & 9,9 & 7,5 & 11 \\
\hline $\begin{array}{l}11: 00- \\
12: 00\end{array}$ & 13,9 & 17,7 & 24,5 & 19,3 & 11,2 & 12,9 & 2,7 & 2,8 \\
\hline $\begin{array}{l}12: 00- \\
13: 00\end{array}$ & 15,8 & 12,4 & 24,1 & 23,7 & 19,3 & 22,1 & 8,2 & 6,9 \\
\hline $\begin{array}{l}13: 00- \\
14: 00\end{array}$ & 5,1 & 5,1 & 0,7 & 1,1 & 3,1 & 2,4 & 7 & 8,5 \\
\hline $\begin{array}{l}14: 00- \\
15: 00\end{array}$ & 14 & 16,3 & 3,3 & 2,5 & 18,3 & 17 & 19,2 & 16,7 \\
\hline $\begin{array}{l}15: 00- \\
16: 00\end{array}$ & 15,3 & 16,3 & 15,3 & 14,6 & 11 & 10,4 & 23,3 & 19,2 \\
\hline $\begin{array}{l}16: 00- \\
17: 00\end{array}$ & 8,9 & 8,4 & 5,7 & 14 & 16,9 & 16,6 & 13,9 & 14,8 \\
\hline $\begin{array}{l}17: 00- \\
18: 00\end{array}$ & 9,4 & 8,2 & 3,5 & 8,5 & 9,6 & 8,8 & 18,1 & 20,1 \\
\hline
\end{tabular}

The analysis of data on the mental state and keyboard handwriting makes it possible to conclude the influence of each lighting mode on the participants.

1. The lighting mode with a color temperature of $4000 \mathrm{~K}$ and an illuminance of 300 lux was found to be the most optimal mode for the subjects. The subjects had consistently high indicators for the number of characters and a tiny number of errors when entering text compared to the other three weeks. The better values of these indicators were recorded at 10:00, 13:00 and gradually decreased by the end of the day.

2. The performance and activity levels were consistently high in the first half of the day in the second week using the lighting mode of $6100 \mathrm{~K}$ and 675 lux. At the same time, the total number of characters increased compared with the first week. It is worth noting that the number of errors 
remained at the same level as in the first week. Some participants reported increased productivity under this mode but an unsatisfactory mental state. Irritability, anxiety and significant fatigue by the end of the day was also observed.

3. The levels of performance, emotional state, and activity were low in the third week (mode 2700 $\mathrm{K}$ and 275 lux). In addition, the total number of errors increased when compared with the first week of the experiment. Also, the amount of the text entered decreased when compared with the first week. This mode has a negative impact on the mental state and performance of individual participants. Participants noted drowsiness and reduced motivation to work.

4. The subjects maintained consistently high indicators of mental state and the level of performance in the fourth week. Schedule of changes in lighting modes: 10:00-13:00 - Relaxation $3500 \mathrm{~K}$ and 325 lux, 13:00 - 14:00 - Standard, 14:00-18:00 - Activation 6100 K and 675 lux. The subjects typed a large number of characters while making a small number of errors when entering text in the afternoon under this mode. The smallest number of errors is observed from 10:00 to 14:00, compared with all previous weeks. At the same time, the subjects experienced problems with sleep, felt tired, and emotional instability by the end of the day.

\section{Discussion}

One of the main results of the study is the obtained dependences describing the subject's reactions under illumination with different photometric characteristics. When designing adaptive lighting systems, as the most effective systems for improving the performance and mood of a subject, all technical solutions should correlate with the user's mental state.

According to the data obtained, it can be argued that the spectral characteristics and the level of illuminance affect the change in the indicators of the subject's mental state, the quality of their sleep, and performance.

With the illumination mode $6100 \mathrm{~K} 675$ lx, the maximum level of activity of the ALS participants is achieved. Also, with this mode, there is a negative effect on sleep. Subjects can feel tired and emotionally unstable towards the end of the day. This mode appears to be resource-intensive for the subjects, but at the same time it increases the level of their productivity. It can be argued about the effectiveness of this mode, hence, more research and developing new techniques are needed to facilitate the subject's activation with a minimum waste of resources.

At $2700 \mathrm{~K} 275$ lx mode, the minimum values are observed for all indicators during the day (low level of assessment of subjective well-being, performance, emotional state). It is not recommended to use this mode as static lighting in an office space in the flow and during the whole day, but a dynamic system is effective at the relaxation stage.

The illumination modes $3500 \mathrm{~K} 325 \mathrm{~lx}$ and $4000 \mathrm{~K} 300 \mathrm{~lx}$ are the most optimal models for the implementation of artificial lighting in adaptive systems for office spaces. These modes allowed to achieve the best results in the indicators of the level of the subject's mental state, the psycho-emotional state, and the subjective assessment of the well-being of workers. Minimal spending of subjects' resources was observed when performing working activities (Kraepelin's arithmetic test, the scheme of the degree of fatigue, the Luscher Color Test).

Future solutions in the field of interior lighting design have to be adapted not only for a specific participant, but also for a group of subjects while improving lighting conditions in any type of space. This adaptive lighting technology can be incorporated into a smart home system, which provides the suitability of its use in residential premises, taking into account suitable lighting scenarios.

The results obtained in this study are a prerequisite for the development of self-learning systems. In the future, such technologies will facilitate the training of lighting systems and developing the user's profile without direct interaction with the system. Developments on creating the subject's profile are already underway at ITMO University [26]. In addition, the study obtained the trail of the keyboard handwriting, audio and video data, which contribute to the algorithm compilation for the future smart lighting system.

\section{Acknowledgements}


This research was realized with the help of the NCCT of and the Creative Lighting Design Department of ITMO University.

The authors would like to express their special gratitude to the head of the NCCT ITMO Oleg Basov, Doctor of Technical Sciences, Professor of the Faculty of Information and Communication Technologies at ITMO University. The authors are also grateful to the team of developers of the ITMO NCCT: Ivan Tolstoy, Egor Aksamentov, Alexander Leonov, Darina Dudina, and Dmitry Podolsky, as well as to Gleb Rogozinsky, the consultant of the NCCT ITMO, Doctor of Technical Sciences, Associate Professor of St. Petersburg State University of Telecommunications named after Prof. MA Bonch-Bruevich.

The package of diagnostic techniques was developed in collaboration with a team of Psychology teachers from St. Petersburg State University. The authors express their personal gratitude to Natalya Vodopyanova, Doctor of Psychology, Professor of the Department of Psychological Support of Professional Activity. The authors express their personal gratitude to Olga Gofman, Candidate of Psychological Sciences, Associate Professor of the Department of Psychological Support of Professional Activity. The authors are also grateful to Vladimir Chesnokov, Ph.D. in Psychology, Associate Professor of St. Petersburg State University.

Luminaires with adjustable color temperature and luminous flux were provided free of charge by PromLed. We would like to express our special gratitude to Ilya Mikhailovich Filippov, PromLED Development Engineer who took an active part in the implementation of the study. The authors would also like to thank all participants who volunteered for the experiments.

\section{References}

[1] D. Pavlov, D. Ivanov and V. Petrov. "The biodynamic energy Efficient lighting for use in scientific and educational institutions", the Second Balkan youth conference coverage 2019 (2019) 1-4. doi:10.1109/BLJ.2019.8883566.

[2] J. Byun, I. Hong, B. Lee, S. Park. "Intelligent household LED lighting system considering energy efficiency and user satisfaction", IEEE Trans. Consum. Electron. (2013) 59, 70-76. doi: 10.1109/TCE.2013.6490243.

[3] Y. Chen, Q. Sun. "Artificial intelligent control for indoor lighting basing on person number in classroom", In Proceedings of the 2013 9th Asian Control Conference (ASCC), 23.06-26.06(2013) 1-4. doi:10.1109/ASCC.2013.6606030.

[4] P. Barrett, F. Davies, Y. Zhang, L. Barrett. "The impact of classroom design on pupils' learning: Final results of a holistic, multi-level analysis", Build. Environ. (2015) 89, 118-133. doi:10.1016/j.buildenv.2015.02.013.

[5] K.C.H.J. Smolders, Y.A.W. de Kort. "Bright light and mental fatigue: Effects on alertness, vitality, performance and physiological arousal", J. Environ. Psychol. (2014) 39, 77-91. doi:10.1016/j.jenvp.2013.12.010.

[6] D. M. Berson, F. A. Dunn, and M. Takao. "Phototransduction by retinal ganglion cells that set the circadian clock,” Science (2002), 295, №5557, 1070-1073. DOI:10.1126/science.1067262.

[7] International Commission on Illumination Commission: Position statement on non-visual effects of light - recommending proper light at the proper time, 2nd edition, 2019. URL: http://cie.co.at/publications/position-statement-non-visual-effects-light-recommending-properlight-proper-time-2nd.

[8] S. Roslyakova et al. "Possibilities to integrate wearable biomonitoring sensors into adaptive lighting systems", IOP Conf. Ser.: Mater. Sci. Eng. (2020) 944012029.

[9] B. Fabio, B. Chiara, L.R. Ornella, B. Laura, F. Simonetta, "Non Visual Effects of Light: An Overview and an Italian Experience", Energy Procedia (2015) 78, 723-728. doi:10.1016/j.egypro.2015.11.080.

[10] N. Wessolowski, H. Koenig, M. Schulte-Markwort, C. Barkmann. "The effect of variable light on the fidgetiness and social behavior of pupils in school", Journal of Environmental Psychology (2014), 39, 101-108. doi:10.1016/j.jenvp.2014.05.001.

[11] C. Barkmann, N. Wessolowski, Schulte-Markwort. "Applicability and efficacy of variable light in schools”, Physiology \& Behavior (2012) 105(3), 621-627. doi:10.1016/j.physbeh.2011.09.020. 
[12] K. Choi, H.-J. Suk. "Dynamic lighting system for the learning environment: Performance of elementary students", Opt. Express (2016) 24. doi:10.1364/OE.24.00A907.

[13] M.E. Kompier, K.C.H.J. Smolders, W.D. van Marken Lichtenbelt, Y.A.W. de Kort. "Effects of light transitions on measures of alertness, arousal and comfort", Physiology \& Behavior (2020) doi:10.1016/j.physbeh.2020.112999.

[14] Smart Lighting Market, Smart Lighting Market by Offering (Hardware: Lights \& Luminaires, Lighting Controls; Software, and Services), Communication Technology (Wired and Wireless), Installation Type, End-use Application, and Geography-Global Forecast to 2025, 2020. URL: https://www.marketsandmarkets.com/.

[15] Netherlands patent №2017111815, 05.08.2015. Nolan Julian Charles, Lawrenson Matthew John [et al.], Resolving lighting preference conflicts, Dutch Patent №. 10.10.2018 Byul. № 28.

[16] US Patent №2011145306/07, 29.03.2010. Klassmann Donald Louis, Murphy Michael Schoen, Intelligent lighting control system, US Patent №. 20.05.2013 Byul. №. 14.

[17]Patent of the Russian Federation №2018145895, 21.12.2018. Gereikhanov R. K, System of adaptive functioning of light-emitting devices, Patent of Russia № 22.06.2020 Byul. № 18.

[18] Netherlands Patent №2017110407, 31.08.2015. Mason Jonathan David, Shraibi Sanae [et al.], Method of controlling a lighting system, computer software product, wearable computing device and a set of lighting systems, Netherlands Patent № 03.10.2018 Byul. №. 28.

[19] Baoshi Sun, Qiaoli Zhang, Shi Cao. "Development and Implementation of a Self-Optimizable Smart Lighting System Based on Learning Context in Classroom", International Journal of Environmental Research and Public Health 21.01.(2020) 17(4), 1217 doi:10.3390/ijerph17041217.

[20] D. Skene, J. Duffy, C. Czeisler. "Effect of Light on Human Circadian Physiology" Sleep Med Clin. (2009) 4(2), 165-177. doi:10.1016/j.jsmc.2009.01.004.

[21] M. Munch, A. Wirz-Justice, S. Brown, T. Kantermann, K. Martin, O. Stefani, S.Vetter et al. "The Role of Daylight for Humans: Gaps in Current Knowledge", Clocks\&Sleep (2020) 2, 61-85. doi:10.3390/clockssleep2010008.

[22] Light and wellness: A circadian approach to lighting design [Electronic resource]. Access mode: https://www.arup.com/perspectives/circadian-approach-to-lighting-design (accessed: 18.12.2020).

[23] K. Choi, C. Shin, T. Kim et al. "Awakening effects of blue-enriched morning light exposure on university students' physiological and subjective responses”, Sci Rep 9, 345 (2019). doi:10.1038/s41598-018-36791-5.

[24] W. Yang, J. Y. Jeon. "Effects of correlated colour temperature of LED light on visual sensation, perception, and cognitive performance in a classroom lighting environment", Sustainability (Switzerland) (2020) 12(10). doi:10.3390/SU12104051.

[25] Production management. Project: human performance and factors of its increase, 2019 URL: http://www.gilpravo.ru.

[26] ITMO Avatar project. An ecosystem of digital assistants for ITMO students and teachers, 2020. URL: https://avatar.itmo.ru/.

[27] Studfile: The technique of "Visual-associative self-assessment of emotional states", 2019. URL: https://studfile.net/preview/9914720/page:64/. 